\title{
El uso de la lengua meta en el curso inicial de Español Lengua Extranjera (E/LE) en Costa de Marfil
}

José Luis Ortega Martín

Universidad de Granada

Bi Drombé DJandué

Lycee Goffry Kouassi Raymond de Sassandra (Costa de Marfil)

Recibido: 27 abril 2012 / Aceptado: 13 enero 2013

ISSN: $1697-7467$

\begin{abstract}
RESUMEN: El uso de la lengua meta no supone el mismo reto en todos los contextos, aunque constituye en todos los casos un factor esencial para la consecución de mejores resultados. Este artículo recoge los principales hallazgos de un trabajo más amplio sobre el uso del español en un contexto de lengua extranjera y en el primer nivel de aprendizaje. El estudio realizado en Costa de Marfil dio resultados muy acordes con las condiciones de aprendizaje en este país, los cuales motivaron a su vez unas cuantas propuestas para aumentar el empleo de la lengua meta en el curso inicial.
\end{abstract}

Palabras clave: lengua meta, lengua materna, producción, exposición

The use of the target language in the initial course of Spanish Foreign Language (S/ FL) in Ivory Coast

ABSTRACT: The use of the target language is not the same challenge in all contexts, although in all cases is essential to achieving better results. This article summarizes the main findings of a broader study on the use of Spanish in a context of foreign language and in the first level of learning. The study was conducted in Ivory Coast and gave results in line with the learning of Spanish in this country. These results led to a few proposals to increase the use of the target language in the initial course.

Keywords: target language, mother tongue, production, exposition

\section{INTRODUCCIÓN}

El uso de la lengua meta ( $\mathrm{Lm})$, como condición determinante en la adquisición de una segunda lengua (L2), es una de las realidades que gozan hoy de la mayor unanimidad en el ámbito de la didáctica de las lenguas. Pero son muchos los obstáculos que dificultan o impiden el total cumplimiento de este requisito en un contexto de lengua extranjera (LE) protagonizado por hablantes no nativos, dificultades relacionadas con los agentes del proceso de enseñanza/aprendizaje (E/A), con los materiales utilizados y con el medio en el cual se lleva a cabo el proceso. Al mismo tiempo, al convertirse el aula en el único lugar de práctica de la lengua por los aprendices, el uso frecuente y efectivo de la Lm en este 
recinto cobra un carácter casi vital y el trabajo del profesor se reviste de una importancia casi mesiánica.

De hecho, lo propio de un aprendizaje en contexto de LE es la total ausencia de la $\mathrm{Lm}$ en las relaciones sociales cotidianas donde otras lenguas tienen todo el protagonismo, aumentándose así la tentación de la lengua materna $(\mathrm{Lm} / \mathrm{L} 1)$, entendida como la inclinación natural de los usuarios de una LE a querer recurrir al idioma que más dominan y que todos comparten en una situación de aula monolingüe para facilitar la comunicación. La tentación de la Lm es más fuerte todavía en el nivel inicial de estudio donde los alumnos debutantes carecen de la suficiente dotación lingüística para utilizar la Lm. Nussbaum (1991) puede afirmar, por eso, que la observación sistemática de clases de LE, sobre todo en niveles de aprendices principiantes, muestra que la $\mathrm{Lm}$ se usa sobre todo como vehículo de regulación de la E/A y de las relaciones afectivas y que, por ello, ocupa un espacio cualitativo y, a veces, cuantitativo superior al de la LE.

La situación del español en Costa de Marfil es esencialmente de LE enseñada en un contexto altamente formal y artificial; corresponde por tanto a lo que se acaba de describir y, en un ámbito escolar regido por el francés como idioma oficial y única lengua de enseñanza, el uso del español se convierte en una conquista diaria y de minuto a minuto. Nos parece, sin embargo, y tal ha sido nuestra hipótesis de partida, que "los profesores marfileños de E/LE pueden utilizar el español con los alumnos principiantes absolutos desde los primeros días de clase para algunas actividades y situaciones, y hacer que estos alumnos noveles vayan participando cada día más en las clases con la Lm como instrumento de trabajo". A pesar de las dificultades de todo tipo, existen, a nuestro ver, factores favorables al uso de la Lm quizá no siempre aprovechados a fondo por parte del profesorado.

El interés del estudio realizado reside en su relativa novedad y en la repercusión que puede tener no solo en la enseñanza del E/LE en Costa de Marfil debido a su carácter empírico, sino también en otros países francohablantes. Si el uso de la Lm en el aula ha sido una preocupación constante a lo largo de la historia de la E/A de las LE, una reflexión sistemática sobre el tema en el contexto marfileño no había sido emprendida todavía, aunque trabajos como los de Koffi $(2009 ; 2010)$ reivindican una mayor atención al lenguaje oral en una enseñanza del E/LE que se pretende comunicativa en Costa de Marfil. Pero la comunicatividad de la lengua no se agota con su oralidad y el lenguaje escrito merece ser atendido también en un contexto de aprendizaje tan formal como el nuestro. Por lo tanto, el uso de la Lm tal como lo hemos planteado se aplica a las dos formas de la lengua y a todas las destrezas lingüísticas.

Ha sido una característica importante del estudio el tener en cuenta a todos los actores de la enseñanza y del aprendizaje del español en nuestro país de acuerdo con la aseveración de Prado Aragonés (2004: 71) según la cual la consecución de resultados satisfactorios en el proceso de E/A lingǘstico y literario necesita que se hallen implicados como agentes de la investigación todos los elementos que intervienen en dicho proceso, especialistas en didáctica, profesores y alumnos, pues ambos pueden hacer importantes aportaciones teóricas y prácticas complementarias e imprescindibles.

Así que los objetivos perseguidos han sido los siguientes:

- dar cuenta de la valoración que tiene entre los profesores marfileños de E/LE, los formadores del profesorado y los alumnos debutantes el uso de la Lm como factor potenciador del aprendizaje; 
- conocer las dificultades que tienen los profesores y los alumnos debutantes para usar el español en clase;

- identificar algunos factores favorables al uso del español en el curso inicial de E/ LE;

- y las estrategias que suelen o pueden utilizar los profesores para hacer posible la comunicación en E/LE con los alumnos noveles.

El conjunto de la información recogida y analizada ha dado pie a propuestas encaminadas a aumentar el empleo del español en los niveles principiantes absolutos de E/LE. Siguiendo en líneas generales las principales articulaciones de la Tesis, presentamos a continuación la situación actual del español en Costa de Marfil, definimos luego el marco conceptual del estudio y describimos a grandes rasgos el proceso de la investigación empírica para, finalmente, exponer los hallazgos y las propuestas que han suscitado.

\section{Situación aCtual del eSPañol en COSTA de MARfil}

Costa de Marfil, ubicada en la parte occidental de África, es el país que alberga el mayor número de estudiantes de E/LE a lo largo y ancho del continente negro. Seguido por Senegal y Camerún, ocupa el sexto lugar en el ranking mundial de los numerosos países interesados hoy en el aprendizaje de la lengua de Cervantes.

La situación del español en esta zona del continente, y concretamente en Senegal y en Costa de Marfil, experimenta un auge sin precedentes y sin comparación posible con el resto de los países africanos. Si se toma como referencia el dato referente a la población de uno y otro país (10 y 18 millones de habitantes respectivamente), resulta aún más sorprendente que sólo estos dos países acaparen el 66\% de la demanda total de español en África. El futuro del español en esta área geográfica concreta se antoja enormemente prometedor, si bien no está exento de retos igualmente ingentes: sin una estabilidad política, económica y social que asegure la calidad de vida de los habitantes de estos países y garantice la continuidad del acceso a la educación a largo plazo, la expansión potencial del español en África seguirá revistiendo un carácter tan frágil como el de la esperanza de vida de la inmensa mayoría de los habitantes de estos países. (López y García, 2007: 57).

Al igual que en todos los países francófonos del África subsahariana, fue el colonizador galo quien, en 1947, introdujo el español en la enseñanza media de Costa de Marfil al trasladar a sus antiguas colonias su mismo sistema educativo. Ello explica también el estudio de otros dos LE como son el inglés y el alemán. Desde su introducción en Costa de Marfil, la evolución de la enseñanza del E/LE se puede analizar desde tres puntos de vista: los libros de texto utilizados en los diferentes niveles de aprendizaje, la metodología aplicada en las aulas y el nivel inicial de estudio. 


\subsection{Los libros de texto utilizados}

Hasta el curso 1997-98, como consecuencia de la influencia de la metrópoli, los libros de texto procedían de Francia ${ }^{1}$. Se caracterizaban por la casi inexistencia de referencias a África, de ahí la distancia cultural entre los contenidos y el alumnado, además de por una imagen de España no muy acorde con la realidad histórica de este país, por lo menos pasados los años 90 .

Los pocos manuales usados en estos países (africanos francófonos), ex-colonias francesas, son los que se utilizan en Francia; la imagen que ofrecen de España es totalmente anacrónica y cargada de tópicos (toros, panderetas...). Sabido es que nuestros vecinos del norte no están por fomentar una visión real y actualizada de unos competidores en el continente africano. (Carrera, citado por Koffi, 2009: 118)

A partir del mencionado curso, se estrenan los manuales Horizontes para todos los niveles de estudio, que corrigen en parte los fallos señalados. Estos libros llevan hoy más de un decenio en nuestras aulas y, sin duda, va siendo hora de cambiarlos. El uso del mismo material durante tanto tiempo representa un peligro para la motivación de sus usuarios más habituales $y$, en ausencia de otras fuentes de formación e información en las localidades del interior del país, mantiene la interlengua del profesor en un letargo que le empobrece y quita eficacia y vitalidad a su docencia.

\subsection{La metodología aplicada}

En cuanto a su adscripción metodológica, desde que comenzó a finales de los años 40, la enseñanza del español en Costa de Marfil siguió las grandes corrientes que marcaron la historia. Hasta la fecha, tres métodos han prevalecido en nuestras aulas: el método tradicional hasta los años 90, un breve entremés audiolingual y, desde finales de los años 90, el método comunicativo (Koffi, 2009: 10). El intento audiolingual, limitado a pocos centros y abandonado pronto por su elevado coste, supuso una temprana preocupación por el uso de la $\mathrm{Lm}$ en el aula, porque este método forma parte de aquéllos que pretenden descartar de plano la intervención de la Lm en el proceso de aprendizaje de una LE: "La traducción está desaconsejada o prohibida porque se parte del convencimiento de que el uso de la lengua materna propicia las interferencias y resta eficacia al aprendizaje de una segunda lengua". (Sánchez, 2009: 75)

La opción por el enfoque comunicativo viene a confirmar esta preocupación y conduce, para empezar, a la adopción de una pedagogía por objetivos (PPO) que se plasma en los manuales Horizontes para, dicen los iniciadores del cambio, "satisfacer las necesidades de aprendizaje de los alumnos por medio de la enseñanza de los actos de habla" (Kouassi,

\footnotetext{
${ }^{1}$ Estos libros de elaboración francesa son, por ejemplo, Tras el Pirineo, Por el mundo hispánico, Siglo XX, ¿Adónde?, ¿Qué tal, Carmen?, Vida y diálogos de España, Lengua y vida, Pueblo I y Pueblo II, Sol y sombra, Caminos del idioma I y Caminos del idioma II y Cambios (Benítez y Koffi, 2010: 255).
} 
2000) (citado por Koffi, 2009: 15). A los objetivos comunicativos recogidos en los nuevos manuales se suman unos objetivos gramaticales para asegurar la adquisición por el alumno de un saber (conocimientos lingüísticos) a poner al servicio de un saber-hacer (aptitudes) y de un saber-ser (actitudes). Pero muy pronto, se considera que había que dar un paso más y se abandona la PPO a favor de una formación por competencias (FPC). La PPO ponía el acento sobre los objetivos lingüísticos y comunicativos a alcanzar; la FPC se centra más sobre el alumno y se le reprocha, entre otras cosas, que no contemple mucho el saber debido a una sacralización del saber-hacer a través del concepto clave de competencia.

\subsection{El nivel inicial de aprendizaje}

La enseñanza del español en Costa de Marfil inauguró el nuevo milenio con una reforma de envergadura: pasar de tercero a primero de secundaria como nivel inicial de aprendizaje. Aunque se está todavía en fase experimental y queda camino por recorrer antes de conseguir todos los objetivos, esta innovación supone nuevos retos para la enseñanza y el aprendizaje del E/LE en nuestro país y, porque toca directamente a los principiantes absolutos, no se podía pasar por alto en este estudio.

La enseñanza del español, gestionada hoy por los nacionales, descansa sobre un cuerpo profesoral esencialmente no nativo. Los profesores de español, formados in situ, vienen directamente de las universidades públicas para ejercer en los numerosos centros privados del país o transitan por la Escuela Normal Superior (ENS) de Abidján para beneficiarse de una formación pedagógica e impartir clases en los centros públicos como funcionarios del Estado. Otro grupo de enseñantes presente en el sector público se compone de estudiantes que, a raíz de una oposición organizada por la misma Función Pública, ha recibido una formación pedagógica intensiva para enseñar español.

Hoy por hoy, la enseñanza del E/LE en Costa de Marfil está marcada por:

- una posición hegemónica del libro de texto en lo que a los materiales didácticos se refiere;

- condiciones materiales y morales de trabajo contaminadas por la corrupción y dificultadas por la antigüedad y la escasez de las infraestructuras y por los efectivos pletóricos;

- una formación continua deficiente al nivel del profesorado en activo;

- y la desmotivación docente debido a la carestía de la vida y a cierto desprestigio de la profesión docente en los últimos años; etc.

En medio de este panorama, el adelantamiento del nivel inicial de aprendizaje es clara señal de esperanza para el futuro del español y de su enseñanza en un sistema escolar donde goza de la preferencia del alumnado por ser de la misma familia latina que el francés. También cuenta con personas formadas y muy amantes del castellano que se dedican a mantener encendida la llama del E/LE en toda la geografía nacional después de más de medio siglo de presencia. Ganada la batalla de la cantidad al nivel africano, estos esfuerzos cotidianos necesitan hoy, para mejorar la calidad de la enseñanza y del aprendizaje, además de posibles apoyos exteriores, el saneamiento del entorno educativo y cierta actualización de la formación docente. 


\section{Marco teórico}

La Lm, opuesta a la lengua de partida, es el nuevo idioma que se pretende adquirir a través de una instrucción formal en la escuela. Ésta remite a la Lm, el francés en nuestro caso, y aquélla a la LE, el español. En esta relación, Lm y LE son las dos caras de una misma medalla, por lo que tratar del uso de la Lm en el aula conlleva siempre una reflexión sobre el lugar y el rol de la lengua de partida. En el fondo, plantear el uso de la LE como recurso didáctico de primer orden infiere que la Lm es un problema en el aula, y aflora entonces una pregunta ineludible: qué hacer de ella ya que, se quiera o no, allí está.

No es de extrañar, entonces, que, como bien lo nota Martín (2000: 15), de entre las cuestiones que han suscitado el interés de los expertos en el tema de las relaciones entre Lm y E/A de otra lengua, se destaquen, entre otras, la conveniencia de la utilización de la L1 en el aula de L2. En efecto, la atención que un determinado método o enfoque presta al uso de la Lm suele expresarse y medirse por su grado de rechazo a la L1 del alumno. Porque la primera referencia metodológica en la historia la constituye un método que se caracterizó, entre otros rasgos definitorios, por el uso preponderante de la $\mathrm{Lm}$ del alumno: el método tradicional (Martín, 2000: 25; Callejón, 2009: 2; Sánchez, 2009: 31).

Las primeras discrepancias con este planteamiento dan lugar a posturas dogmáticas bajo la bandera de los llamados métodos naturales que pretenden hacer que la LE se adquiera en las mismas condiciones que la Lm. De este modo, propuestas metodológicas como el método directo, máximo representante de la corriente naturalista, y otros como el enfoque oral y la enseñanza situacional de la lengua, el método audiolingüístico y el enfoque natural de Krashen y Terrel se posicionan en contra de toda intervención de la Lm y de la traducción en el aula de LE.

Pero todo extremo es negativo. Si el método tradicional no creaba las condiciones idóneas para un aprendizaje eficaz de la LE porque negaba lo esencial de la lengua: su fin comunicativo; el método directo y sus derivados niegan por su parte lo esencial del alumno y, si no es nativo, del profesor: su identidad lingüísticocultural. De hecho, la Lm es uno de los elementos que permite identificar al ser humano. Los seres humanos somos palabras, porque lo que somos profundamente lo tenemos tejido por la lengua que, desde el seno de la madre y el entorno familiar y social, ha venido formando y conformando nuestra visión de nosotros mismos, de los otros y de la vida y del mundo. Y ello explica la inevitabilidad de la Lm en todo aprendizaje, incluso el de una LE.

Trasladada al contexto marfileño de enseñanza y aprendizaje, la inevitabilidad de la Lm se debe a tres fuentes inagotables: los agentes en presencia (el profesorado no nativo y el alumnado), el medio (regido por el francés) y los materiales (huellas de francés en todos los libros de texto). Esta inevitabilidad explica a su vez la utilidad de la L1. Buscar prohibir su presencia puede por lo tanto resultar contraproducente mientras impulsado por un mero capricho metodológico. Lo más razonable, desde nuestro punto de vista, es aprovecharla mientras sea útil para la adquisición de la Lm y dejar que el propio aumento del conocimiento de la LE por parte de los alumnos se encargue de ir haciendo prescindible la Lm. Porque la incomodidad de no poder comunicarse o de hacerlo fatal ayuda menos que una comunicación fluida en Lm puesta al servicio del aprendizaje de la LE.

Desde tal perspectiva, nos parece que bajo los supuestos del enfoque comunicativo se corrigen los dos errores mencionados anteriormente, cometidos el uno por el método tradicional y el otro por el método directo y sus respectivos derivados. Apartándose en efecto 
de los dos extremos arriba reflejados, métodos como la vía silenciosa, la sugestopedia y el aprendizaje comunitario de la lengua muestran cierta flexibilidad con respecto a la presencia de la Lm en el aula, lo cual se adecua sin duda a su carácter humanista y a la importancia que otorgan a los factores afectivos en el aprendizaje de lenguas (Martín, 2000: 36-37).

Estos métodos no han tenido un gran éxito en el mundo, pero con la efervescencia que conoce el enfoque comunicativo desde los años 60-70, parece que estamos en la era de la vía media en la enseñanza de lenguas después de haber experimentado el peligro de los fanatismos metodológicos, por lo menos sobre la cuestión del uso de la Lm. Pero la postura intermedia de esta corriente sobre el tema no puede interpretarse en el sentido de 50 por ciento de Lm y 50 por ciento de LE, sino que ha de entenderse, así pensamos, como una apelación a la creatividad del docente para buscar siempre la manera de ofrecer al alumnado un input comprensible y masivo y recurrir a la Lm en caso de extrema necesidad para poner en marcha la comunicación en el aula.

El uso de la Lm es avalado por tres teorías como imprescindible para la adquisición de cualquier LE: la teoría del input comprensible (Krashen, 1985) que enfatiza la exposición a la lengua (escuchar/leer), la teoría del output comprensible (Swain, 1985) que resalta el papel de la producción (hablar/escribir) (citados por Arnaiz y Peñate, 2004) y la teoría de la interacción en sus vertientes social (Long, 1980) y cognitivo (Pica, 1989; 1994) que aboga por la negociación del significado en situaciones reales de comunicación (citados por Barranco, 2007). Se puede ver entre estos tres componentes del uso lingüístico una relación casi "trinitaria": en las condiciones normales de comunicación, en efecto, la recepción nace de la producción y de ambas procede la interacción. Para aprender eficazmente una LE, el sujeto tiene que estar en contacto permanente con ella a través de la escucha y la lectura, pero también necesita arriesgarse con ella hablando y escribiendo para intentar siempre acortar la distancia entre su interlengua y la Lm, de ahí la utilidad de la interacción comunicativa con un nativo o, al menos, con un interlocutor más conocedor del idioma.

Así las cosas, el papel de facilitador del aprendizaje asignado al profesor supone por su parte animar a los alumnos a usar la Lm y facilitarles tal uso a través de sus conductas y actitudes, de la metodología que utiliza, de los materiales que emplea y de las actividades que propone. Aprender una lengua es aprender a usarla y los que más precisan escuchar, hablar, leer, escribir e interactuar con esta lengua son los que la aprenden. El quehacer didáctico y pedagógico se cifra por lo tanto en dos tareas esenciales: proporcionar el input suficiente y necesario y crear las condiciones idóneas para que los discentes se animen a producir cantidad de output. En un contexto de LE, ello se puede lograr diversificando las fuentes de input, variando las actividades, fomentando la interacción alumno-alumno y creando y manteniendo un ambiente grato y motivador.

\section{Diseño experimental}

Hemos realizado un estudio descriptivo de tipo cuantitativo con un componente cualitativo introducido a conciencia para tener en cuenta el carácter subjetivo de la realidad educativa que, como realidad social y humana, no siempre es expresable en números y porcentajes. Así pues, de los 29 variables manejados, 22 han recibido un tratamiento cuantitativo a través 
del Programa SPSS (análisis descriptivo e inferencial) y solo 7 han recibido un tratamiento cualitativo. Se trata por tanto de un método mixto de investigación con un caso de doble triangulación que, además de combinar los métodos cuantitativo y cualitativo, ha utilizado tres fuentes de datos: el alumnado, el profesorado y los formadores del profesorado.

Ha prevalecido en la selección de la muestra dos criterios no probabilísticos de selección: el muestreo por cuota que intenta obtener representantes de los diversos elementos de la población total en las proporciones en que se presentan allí (Cohen y Manion, 2002: 138) y, dentro de cada componente de la población meta, el muestreo no probabilístico casual en el que el criterio de selección de los individuos depende de la posibilidad de acceder a ellos (Ortega y Madrid, 2009: 188). Así es como hemos encuestado a 1589 alumnos, 98 profesores y 14 formadores del profesorado en 20 ciudades de Costa de Marfil y en no menos de 41 centros escolares.

Para cada componente de la muestra, hemos concebido un cuestionario diferente para tener en cuenta su peculiar relación con el tema tratado. Han sido cuestionarios mixtos en todos los casos con preguntas cerradas, preguntas abiertas y preguntas semi-cerradas. Elaborados los cuestionarios a partir de modelos ofrecidos por Madrid (1999: 101-102) y Aurrecoechea (2002: 49-51), se inició un proceso de validación de los mismos, sometiéndolos a la evaluación de tres especialistas.

De las tres encuestas realizadas en el país de estudio, solo nos hemos encargado de la encuesta dirigida a los formadores del profesorado por la imposibilidad de estar fuera de España el tiempo necesario para administrar todos los cuestionarios a una muestra importante. En cuanto al cuestionario del alumnado, debido al nivel de estudio elegido, lo primero ha sido traducirlo al francés para asegurar que los alumnos entiendan bien las preguntas y respondan con la máxima precisión posible. Esta precaución aseguraba, además, que expresándose en su L1, dieran respuestas más personales a las preguntas planteadas.

Esta encuesta, como la dirigida a los profesores, se ha aplicado en 2010 y en 2011. En 2010, el cuestionario había sido enviado por correo electrónico directamente a los profesores colaboradores o a un coordinador. Éste los había imprimido y repartido entre los diferentes profesores a él indicados. En 2011, nos hemos beneficiado también de la disponibilidad del mismo coordinador para multiplicar el cuestionario y repartirlo entre otros profesores. Como este año no hemos podido estar en el país de estudio por la situación política, los cuestionarios respondidos nos han llegado por correos. Una crisis postelectoral iniciada en noviembre de 2010 había cuLminado en guerra civil a finales de marzo de 2011. Este acontecimiento perturbó la evolución del trabajo al impedir la aplicación de los últimos cuestionarios y al retrasar el envío de los cuestionarios respondidos.

\section{Conclusiones y PROpuestas}

Los alumnos meta son chicos y chicas de 10 a 19 años que estudian E/LE como principiantes absolutos en un país donde se habla francés y más de 60 lenguas autóctonas. Proceden de centros públicos y privados de la enseñanza media, tanto de Abidján, capital económica, como de ciudades más modestas. El aprendizaje se desarrolla en aulas con efectivos pletóricos (más de 50 alumnos en general). Por cursar dos niveles distintos debido a un adelantamiento del estudio del español que tarda aún en generalizarse y acabar con 
el antiguo modelo, se ha distinguido entre APA (antiguos principiantes absolutos) y NPA (nuevos principiantes absolutos) respectivamente de tercero y primero de secundaria. Los profesores informantes son hombres y mujeres con una experiencia docente de 0 a 25 años, que imparten español en centros públicos y privados en Abidján o en ciudades del interior. La mayoría sabe manejar las TIC y tiene una actitud positiva hacia el primer curso de E/ LE. Los formadores del profesorado son profesores en la ENS encargados de la formación inicial de los futuros profesores o especialistas de la Pedagogía encargados de la formación continua de los profesores en ejercicio, con una experiencia docente de 3 a 26 años.

De cara a la valoración del uso de la $\mathrm{Lm}$ en el aula, los resultados han reflejado la tradicional pugna entre profesores y formadores sobre la exigencia de usar el español en clase. Sobre esta cuestión, los primeros suelen tachar a los segundos de "idealistas" desconectados de la realidad de las aulas y que, desde el confort de sus despachos, dictan normas y recetas pedagógicas imposibles de aplicar. Así que los formadores ven más importante, más motivador para los alumnos y menos difícil que los profesores el uso de la $\mathrm{Lm}$ en el aula. Pronunciándose sobre la importancia y el placer de usar el español en clase, tanto los APA como los NPA han tenido opiniones muy positivas, entendiendo, sin embargo, que les pueden ser beneficiosos usos ocasionales del francés.

En relación a las dificultades de los alumnos para utilizar el español, los resultados son característicos, en su conjunto, de niveles iniciales donde los sujetos carecen del suficiente conocimiento para mantener una comunicación rica. Desde el punto de vista de la organización de la clase, se ha podido observar una predominancia de las actividades en grupo-clase sobre las actividades en pequeños grupos. Además, las habilidades más difíciles de desarrollar son aquellas que exigen una mayor implicación personal a nivel cognitivo y psicológico y/o aquellas que suponen una mayor exposición a los ojos y oídos de los compañeros y del profesor. Hablar se ha destacado por eso como la destreza más costosa independientemente de los factores diferenciadores considerados (nivel, edad, sexo, centro); y escuchar la habilidad más fácil de desarrollar en la mayor parte de los casos. Las dificultades de los profesores en el primer nivel de estudio son diversas: entablar una comunicación fluida con los alumnos noveles, falta de materiales didácticos, efectivos pletóricos, una formación continua deficiente y poco estructurada y algunos fallos del sistema educativo relativos a la escasez del tiempo de aprendizaje o a la imposición de un esquema para el estudio de textos. También se ha dejado claro que el vocabulario abstracto y la gramática son los contenidos más difíciles de explicar en el curso inicial. Por su parte, ciertos formadores han apuntado a la propia competencia de los docentes en la lengua que enseñan.

El tercer objetivo de la investigación se ha dedicado a descubrir factores propicios al empleo del español. Los resultados obtenidos han revelado tres elementos susceptibles de ayudar a un uso temprano del español en el curso inicial: la fuerte implantación del francés en la sociedad marfileña pese a la vitalidad de las numerosas lenguas locales, el gran aprecio que tienen los alumnos por el español y el hecho de que muchos inicien el estudio del nuevo idioma teniendo ya algunas nociones del mismo. Estos antecedentes, cuantitativamente insignificantes en sí, solo tienen un valor afectivo en la medida en que pueden instalar al alumno novel en una predisposición favorable para aprender una lengua por la que se interesaba ya de una manera u otra. El uso de la Lm se ve favorecido así por el hecho de partir del francés como L1 gracias al parentesco lingüístico entre el español y el francés. Un elemento en el que se materializa dicho parentesco es el común uso del sistema alfabético latino, que constituye para el profesor y el alumno novel un punto de arranque muy favorable. 
La referida pugna entre profesores y formadores puede achacarse a que a los primeros no se les dice exactamente lo que tienen que entender por uso de la $\mathrm{Lm}$. Se les recomienda usar el español en clase sin aclararles lo que ello quiere decir, dejándoles suponer, como nos han dado a leer algunos, que ello significa realizar todas las actividades en español sin recurrir nunca al francés, de allí su reticencia o rebeldía. Porque, aunque algunos docentes entienden que usar el español en clase no significa desterrar la Lm del proceso, los hay que lo entienden como el total rechazo al francés. Dicho esto, al tenor de la gran importancia que le otorgan al empleo de la Lm a pesar de las dificultades, los profesores piensan que el momento ideal para utilizar el español es desde el primer día de clase. Pero los docentes de centros privados han expresado una opinión diferente de la de sus colegas de centros públicos. Al encontrar más difícil el uso de la Lm en el curso inicial, se han mostrado también menos partidarios de una temprana instauración del español como instrumento de comunicación.

Las propuestas hechas por los formadores apuntan ante todo a mejorar la formación continua del profesorado, debido a que consideran el mismo nivel lingüístico de los profesores como una de las principales causas de la gran presencia del francés en nuestras aulas de E/ LE. Conscientes además de la influencia de las condiciones físicas y materiales de trabajo, proponen que se construyan nuevos centros para reducir los efectivos y que se permita a los institutos conseguir laboratorios de lengua. En cuanto a estrategias utilizables para aumentar el caudal de LE en la clase, se sugiere a los profesores escuchar a hablantes nativos del español a través de la radio, del internet o de cintas, leer mucho en la lengua que enseñan, practicar juegos didácticos y valorar las notas de participación de los alumnos, preparar sus clases con esmero y ser pacientes con los alumnos, etc.

Llegado a este punto, hemos encontrado que el conjunto de los resultados obtenidos muestra que existen recursos para que los profesores marfileños de E/LE utilicen el español con los principiantes absolutos desde los primeros días de clase para algunas actividades y situaciones, y hagan que los alumnos noveles vayan participando cada día más en las actividades con la Lm como instrumento de trabajo. A pesar de las dificultades, que siempre hay y habrá, existen factores favorables a nivel afectivo y cognitivo para aprovechar. El aprovechamiento de dichos factores no deja, sin embargo, de ser harina de otro costal y, no cabe duda que en muchos casos dificultades relacionadas a la competencia del profesor y a las condiciones de trabajo pesen más a la hora de usar la Lm en el aula. Pero las opiniones de los actores en presencia quedan insuficientes para comprobar esta realidad; hacen falta observaciones directas o grabaciones de clases para analizar informaciones concretas, sin duda más reveladoras de lo que ocurre en nuestras aulas de E/LE.

Sobre la base de estos resultados, pero también de nuestra propia experiencia como profesor de E/LE en el país de estudio, hemos hecho propuestas para aumentar el flujo de Lm en el curso inicial, siguiendo una evolución desde fuera hacia dentro del aula.

Fuera del aula, es recomendable introducir la didáctica en nuestras universidades públicas para que desde allí puedan beneficiarse de una formación inicial todos aquellos que enseñan español en los centros privados con sus únicos conocimientos académicos. Y que se incluya en la formación inicial de los futuros profesores conocimientos teóricos y prácticos que garanticen la plena asunción por ellos mismos de su propia formación continua, porque la mejor manera de formar a futuros profesores de calidad es formar a futuros investigadores. La investigación de la propia docencia es una técnica eficaz de reflexión 
sobre la propia praxis que, si se lleva a cabo con la debida constancia y seriedad, redunda forzosamente en una mejora de la enseñanza y del aprendizaje. Además, urge replantear en Costa de Marfil la evaluación docente, porque una evaluación acertada del profesorado es aquella que contempla sus actuaciones dentro del aula, escenario por excelencia del quehacer docente. Y quienes mejor pueden apreciar estas actuaciones son los propios alumnos y no el superior jerárquico que nunca está en el aula. Proponemos entonces sumar al actual modelo de evaluación desde arriba un modelo de evaluación desde abajo para abarcar más aspectos de la profesión docente, especialmente el uso de la $\mathrm{Lm}$ en clase en lo que toca a los profesores de LE.

Dentro del aula, hemos propuesto una mejor gestión didáctica y pedagógica de las primeras lecciones, estos contenidos básicos que se imparten antes de pasar a utilizar el libro de texto en el curso inicial, y cuya elección, selección y programación dependen de cada profesor. Otra propuesta es que el profesor y sus alumnos puedan hacer el aula más expresiva y participativa en el proceso de aprendizaje usando letreros, pósteres o carteles en español, dibujos o símbolos relacionados con el mundo hispano, un refrán o un dicho que se escribe con letras grandes y coloreadas en un rincón de la pizarra o en un papel-cartón que se coloca durante un tiempo. Además, en nuestras aulas con efectivos pletóricos, los trabajos en pequeños grupos tendrían la ventaja de permitir a cada alumno hacer algo con la Lm. El uso razonado de juegos y soportes didácticos es también aconsejable para sacar partido de dos elementos importantes en todo aprendizaje: el espíritu lúdico y la vista como medio privilegiado de conexión con el mundo exterior. Hemos terminado proponiendo actividades casi ausentes en nuestras aulas para fomentar la variación y desarrollar harmoniosamente las cuatro destrezas lingüísticas en el alumno debutante. Es evidente que todas estas propuestas requieren de unas buenas condiciones físicas y materiales de trabajo, y le toca al Estado marfileño, en última instancia, mejorar dichas condiciones edificando más infraestructuras para la educación.

\section{REFERENCIAS BIBLIOGRÁficas}

Arnaiz, P. y Peñate, M. (2004). "El papel de la producción oral (output) en el proceso de aprendizaje de una lengua extranjera (LE): el estudio de sus funciones", en Porta Linguarum, 1: $37-59$.

Aurrecoechea, E. (2002). La pronunciación. Su tratamiento en el aula E/LE. Memoria de Máster. Madrid: Universidad Nebrija.

Barranco, J. L. (2007). La influencia de las modificaciones conversacionales del profesor en la reestructuración del conocimiento lingüístico de los aprendices: un estudio de caso. Memoria de Máster. Barcelona: Universidad de Barcelona.

Benítez, S. G. y Koffi, K. H. (2010). "La situación del español en costa de marfil: contextos específicos para la enseñanza de ELE", en Monográficos marcoELE, 11: 252-263.

Callejón, L. (2009). "Hacia un método eficaz de enseñanza de lengua extranjeras", en Innovación y Experiencias Educativas, 16: 1-8.

Cohen, L. y Manion, L (2002). Métodos de investigación educativa. Madrid: La Muralla.

Koffi, K. H. (2009). Panorama de la pluralidad lingüistica y cultural de Costa de Marfil: situación del español como Lengua extranjera. Memoria de DEA. Granada: Universidad de Granada. 
Koffi, K. H. (2010). La didáctica de la competencia comunicativa oral en el aula de E/LE en Costa de Marfil: problemas y perspectivas. Tesis Doctoral. Granada: Universidad de Granada.

López, M. y García, C (2007). "El español en Senegal, Costa de Marfil, Burkina Faso, Cabo Verde y Gambia", en Anuario del Instituto Cervantes 2006-07: 57-62. Disponible en http://cvc.cervantes.es/lengua/anuario/anuario_06-07/pdf/paises_05.pdf. Consultado el 16 de diciembre de 2009.

Madrid, D. (1999). La investigación de los factores motivacionales en el aula de idiomas. Granada: Grupo Editorial Universitario.

Martín, J. M. (2000). La lengua materna en el aprendizaje de una segunda Lengua. Sevilla: Universidad de Sevilla, Secretariado de Publicaciones.

Nussbaum, L. (1991). "La lengua materna en clase de lengua extranjera: entre la ayuda y el obstáculo", en Signos Teoría y Práctica de la Educación, 4: 36-47.

Ortega, J. L. y Madrid, D. (2009). “¿Qué técnicas de comunicación oral valoran más los profesores y alumnos en la clase de inglés", en Porta Linguarum, 12: 183-204.

Prado, J. (2004). Didáctica de la lengua y la literatura para educar en el siglo XXI. Madrid: La Muralla.

Sánchez, A. (2009). La enseñanza de idiomas en los últimos cien años. Métodos y enfoques. Madrid: SGEL. 